\title{
Erratum: Large Unidirectional Magnetoresistance in a Magnetic Topological Insulator [Phys. Rev. Lett. 117, 127202 (2016)]
}

\author{
K. Yasuda, A. Tsukazaki, R. Yoshimi, K. S. Takahashi, M. Kawasaki, and Y. Tokura
}

(A) (Received 31 March 2019; published 18 April 2019)

DOI: 10.1103/PhysRevLett.122.159903

In Eq. (2) of the Letter, we derived the expression of UMR based on the Boltzmann transport theory. In the equation, the integration is taken over $k_{x}$, the momentum along the $x$ direction. Here, the derivative symbol $d$ was missing after the integral. Namely, Eq. (2) should be corrected as

$$
\Delta R_{\mathrm{xx}} \propto j_{x} \int d k_{x}\left(-\frac{1}{\tau^{+}}+\frac{1}{\tau^{-}}\right)\left(\frac{\partial^{2} f}{\partial E^{2}}\right) .
$$

This typo does not alter any conclusions drawn in the paper. 